BIOTROPIA NO. 16, $2001: 28$ - 38

\title{
CHARACTERIZATION OF MALAYSIAN WILD BANANAS BASED ON ANTHOCYANINS
}

\author{
MUHAMMADASIF JAVED*, MAK. CHAIANDROFINAYASMINOTHMAN \\ Division of Genetics, Institute of Biological Sciences, University of Malaya \\ 50603 Kuala Lumpur, Malaysia
}

\begin{abstract}
The male buds of 16 Musa species (Musaceae) populations were investigated by HPLC for the occurrence of anthocyanins. The investigation was based on the presence of 6 anthocyanins. The 16 Musa samples could be classified into three distinct species i.e. Musa acuminata, Musa violascens and Musa balbisiana. Musa acuminata could be divided into two subspecies : malaccensis (lowland) and tmncata (highland) according to their constituents and content of major anthocyanins. No variation was observed in the composition of the anthocyanins of Kedah type ssp. siamea and Selangor types ssp. malaccensis. The classification of $M$. acuminata into two subspecies based on anthocyanin data further supported the current taxonomic grouping of the species.
\end{abstract}

Key words: Musa acuminata/Musa violascens/Musa balbisiana/Musaceae /HPLC /chemotaxonomy

\section{INTRODUCTION}

The classification of the genus Musa as proposed by Cheesman (1947) is the only one which accords satisfactorily with the known taxonomic and cytogenetic facts. Of the four named sections, Eumusa is the best known partly because the plants are hardy and fairly easy to grow whereas the Callimusa is the least known section. Section Eumusa is the most important as the progenitor of all cultivated bananas. Musa acuminata (AA) and Musa balbisiana (BB) belong to this section. Among the cultivated bananas, different cultivars are classified according to their genomic constitution and ploidy level.

Malaysia is one of the centers of diversity of wild and cultivated bananas. Simmonds (1955) reported four species from the Malaysian peninsula. The two species Musa violascens and Musa gracilis are ornamental wild bananas whereas Musa acuminata and Musa balbisiana are the two most important species of the genus Musa. Musa acuminata is the most variable species of the two in Malaysia being the progenitors of several local diploid and triploid banana cultivars. Three different subspecies of Musa acuminata suggested (Simmonds 1955) were ssp. malaccensis (Selangor), truncata (Cameron highland) and siamea (Kedah). This classification was questioned by Hari (1968) and then later on Shepherd (1988) based on chromosomal translocations. Therefore, the nomenclature of local wild Musa species has been complicated by taxonomic revisions. Recent renewed interest

* Corresponding Author: e-mail. J95asit@umcsd.um.edu.my rocketmail. Asif_Javed@rocketmail.com 
in the utilization of wild banana sources had further elucidated the need to characterize different wild Musa species in Malaysia.

Anthocyanins constitute the most important group of plant pigments. In 70 species of 33 families of angiosperms, they were found in membrane-bounded anthocyanoplasts located within the main cell vacuole. Apart from contributing cyanic color to flower and fruits, the pigments also play an important role during pollination, and are of great economic and genetic importance. Previous surveys of anthocyanins in the bracts of banana have been reviewed (Simmonds 1954 b,c; Horry and Jay 1988 a,b). The distribution of various anthocyanidins among different banana cultivars gave good support to the taxonomic ordering within the genus (Horry and Jay 1988a,b).

The present study described the relationship between different wild bananas of Malaysian origin based on the distribution of anthocyanins.

\section{MATERIALS AND METHODS}

\section{Sample Collection}

Wild Musa species samples were collected from different parts of Peninsular Malaysia. The types and number of samples collected and their locations are shown in Table 1. Fourteen populations of wild Musa acuminata were sampled. Samples of Musa violascens and Musa balbisiana were also selected for comparative studies. Ten suckers each of Musa acuminata, Musa violascens and Musa balbisiana were collected from different mother plants and these were planted in the university farm for evaluation. Suckers were planted in holes of 30-40 cm deep, spaced at $2 \mathrm{~m} \mathrm{x} 2 \mathrm{~m}$. At planting, organic compost was applied in the holes. Weeding was done manually.

\section{Anthocyanin Extraction}

Variation in anthocyanin pigments was studied from male buds collected from 16 samples (Table 1). Each banana sample consisted of three male buds as three replicates. The outermost male bracts were used for anthocyanin extraction by grinding 4-5 g of bracts to powder form in liquid nitrogen. Anthocyanin pigments were then extracted by boiling the powder in $40 \mathrm{ml}$ of $\mathrm{MeOH}$ - EtOH (1:1) for 40 minutes. Crude extracts were concentrated to $5 \mathrm{ml}$, and filtered through glass wool (Horry and Jay 1988a). Filtrates were stored at $-70^{\circ} \mathrm{C}$ till used for thin layer chromatography (TLC) and high pressure liquid chromatography (HPLC).

\section{TLC and HPLC analysis}

Thin layer chromatography (TLC) was carried out on $0.1 \mathrm{~mm}$ cellulose plates (Merck. 1.05716. 20x20 cm). Development was accomplished with solvent mixtures prepared from analytical grades of hydrochloric acid, formic acid and deionized dis- 
Characterization of Malaysian wild bananas - Muhammad Asif javed et al

Table 1. Samples of wild Musa species

\begin{tabular}{|c|c|c|c|c|c|}
\hline No & $\begin{array}{l}\text { Local } \\
\text { name } \\
\text { /code }\end{array}$ & Botanical name & Location & State & Form \\
\hline 1 & RI & Musa acuminata & Kajang & Selangor & Selangor \\
\hline 2 & IPTJ & Musa acuminata & $\begin{array}{l}\text { University of Malaya } \\
\text { campus }\end{array}$ & Selangor & Selangor \\
\hline 3 & BD1 & Musa acuminata & Damansara highway & Selangor & Selangor \\
\hline 4 & BD2 & Musa acuminata & Sungei Buloh & Selangor & Selangor \\
\hline 5 & $\mathrm{BCl}$ & Musa acuminata & Genting foothills & Pahang & Selangor \\
\hline 6 & $\mathrm{BC} 3$ & Musa acuminata & Genting $2000 \mathrm{~m}$. & Pahang & Cameron highland \\
\hline 7 & Segun & Musa acuminata & Kampung awah & Pahang & Kedah type \\
\hline 8 & Flava & Musa acuminata & Pulau Tijau & Pahang & Kedah type \\
\hline 9 & Kra & Musa acuminata & Karak highway & Pahang & Selangor \\
\hline 10 & Perak & Musa acuminata & Grik & Perak & Selangor \\
\hline 11 & PPC & Musa acuminata & Tapah & Perak & Selangor \\
\hline 12 & Rangis & Musa acuminata & Jeram & Kelantan & Kedah type \\
\hline 13 & $J-4$ & Musa acumtinata & Jeli & Kelantan & Selangor \\
\hline 14 & Sintok & Musa acuminata & Sintok & Kedah & Selangor \\
\hline 15 & $\mathrm{BC} 2$ & Musa violascens & Genting & Pahang & Species \\
\hline 16 & Gala & Musa balbisiana & Kuala terengganu & Terengganu & Species \\
\hline
\end{tabular}

"tilled water. Two solvent systems used for TLC were : a. System I. Cone. HC1 Formic acid - Water (24.9:23.7: 51.4), b. System II. Cone. HC1 - Formic acid Water (7.1:31.4:61.5).

Developing tanks were lined with heavy grade filter paper and allowed to equilibrate overnight prior to use. TLC plates were kept at $80^{\circ} \mathrm{C}$ overnight. A base line was drawn on the loading end and a 1-2 ul of concentrated anthocyanin solutions were loaded. There were 10 samples loaded on each plate. Rf values taken for each pigment according to their mobility on the TLC plate were calculated as follows;

$$
\mathrm{Rf}=\frac{\text { Distance of migration substance }}{\text { Distance of migration of solvent front }}
$$

HPLC analysis of anthocyanin pigments was carried out on a Millipore Waters $^{\mathrm{TM}}$ liquid chromatograph equipped with a variable wavelength Waters ${ }^{\mathrm{TM}} 486$ detector, Waters $^{\mathrm{TM}} 600$ controller and Waters $^{\mathrm{TM}} 717$ plus auto-sampler was used. Anthocyanin pigments separation was carried out using reverse phase C - 18, Inertsil ODS -2 (4.6 x $1.6 \mathrm{~mm}) 5$ urn column (Horry and Jay 1988a).

HPLC was run according to Horry and Jay (1988a), with slight modifications. Two solvents used were 5\% Formic acid and absolute methanol (5:95, v/v). Concentrated anthocyanin extracts were diluted ten times with $0.1 \mathrm{~N} \mathrm{HC1}$, and filtered just before injection. Flow rate was maintained at $0.80 \mathrm{ml} / \mathrm{min}$ with an injection volume of $20 \mathrm{ul}$. Detection was observed at $514 \mathrm{~nm}$ or 240 to $600 \mathrm{~nm}$ with scanning detector for spectral identification of the peaks. The aglycone moiety of the 
anthocyanin for each HPLC peak was identified according to the maxima of absorbance in the visible range (compounds 3, 6, 8: $X_{\max \text { vis }}=530,535,535 \mathrm{~nm}$; compounds 4, 5, 7: A. max v $\mathrm{j}_{\mathrm{s}}=520,522,522 \mathrm{~nm}$ ) and to their retention times whereas glycosidic pattern was determined by TLC. The composition of the two solvents, flow rate and time are given in Table 2.

Table 2. Method used for anthocyanin separation by HPLC system (Horry and Jay 1988a)

\begin{tabular}{ccccc}
\hline Event & Time (min) & Flow rate (ml) & 5\% Formic acid (\%) & Absolute methanol (\%) \\
\hline 1 & 0 & 0.80 & $80 \%$ & $20 \%$ \\
2 & 20 & 0.80 & $55 \%$ & $45 \%$ \\
3 & 25 & 0.80 & $20 \%$ & $80 \%$ \\
4 & 28 & 0.80 & $20 \%$ & $80 \%$ \\
5 & 29 & 0.80 & $80 \%$ & $20 \%$ \\
6 & 30 & 0.80 & $20 \%$ & $80 \%$ \\
\hline
\end{tabular}

The peaks showing different anthocyanin pigments (delphinidin, derivative of cyanidin, cyanidin, petunidin, peonidin and malvidin) were used for sample comparison.

\section{Chemometric Analysis}

HPLC data from 16 Musa samples were subjected to cluster analysis and principal component analysis (PCA) using SPSS statistic program (SPSS, Inc.). Relative amounts of the anthocyanins expressed by percentages were used as an input matrix.

\section{RESULTS}

Anthocyanins were extracted from the outer most bracts tested through thin layer chromatography (TLC) followed by high pressure liquid chromatography (HPLC). Different pigments separated by HPLC were determined according to increasing retention times, and only six compounds were identified. The absence of a shoulder around $330 \mathrm{~nm}$ indicated the absence of acylation. The presence of a shoulder in the visible spectrum at $440 \mathrm{~nm}$ and the Rf values obtained on TLC indicated the presence of 3- rutinosides as also reported by Horry and Jay (1988a).

Thin layer chromatography on $0.1 \mathrm{~mm}$ cellulose layer plates clearly showed the separation of different anthocyanin pigments. Rf values obtained on TLC indicated the presence of 3- rutinosides namely 3-O-rutinosyldelphinidin, 3-O-rutinosylcyanidin, 3-O-rutinosylpeonidin, and 3-O-rutinosylmalvidin (Table 3).

High pressure liquid chromatography (HPLC) of anthocyanins resolved Musa samples into a maximum of nine peaks. The peaks appearing between 10 to 21 minutes were the six peaks representing the six anthocyanins according to their retention times (Horry and Jay 1988a,b). 
The same sample injected three times did not show any difference in the chromatograms showing the stability of the system and the anthocyanin pigments. Similarly, anthocyanin components extracted from the three outermost bracts from the same male bud did not show any difference in the number of anthocyanin components. The color of the bract in the male inflorescence progressively vanishes from the outside bract (bract 1, the oldest) to the inner bracts. It was observed that the relative distribution of the anthocyanin pattern is stable from the outer bracts to the inner bracts.

Extracts from Musa acuminata accessions, BC2 and Gala were analyzed and the results are presented in Table 4. Six previously reported anthocyanins were observed to be piesent in different combinations and percentages among all the samples studied except Flava (Table 4).

Table 3. Anthocyanin of banana bracts separated through TLC by using two different solvent systems

\begin{tabular}{|c|c|c|c|c|c|}
\hline \multirow{2}{*}{$\begin{array}{l}\text { Compound } \\
\text { number }\end{array}$} & \multirow{2}{*}{ Assigned structure } & \multirow{2}{*}{ Abbreviation } & \multirow{2}{*}{$\begin{array}{l}\text { HPLC retention } \\
\text { times (min) }\end{array}$} & \multicolumn{2}{|c|}{$\begin{array}{c}\text { Observed Rf } \\
\text { values }\end{array}$} \\
\hline & & & & Syst. 1 & Syst. 2 \\
\hline 1 & 3-O-Rutinosyldelphinidin & $\mathrm{De}$ & 12.43 & 0.32 & \\
\hline 2 & Cyanidin derivative & Cyd & 13.30 & & \\
\hline 3 & 3-O-Rutinosylcyanidin & $\mathrm{Cy}$ & 15.51 & 0.26 & 0.50 \\
\hline 4 & 3-O-Rutinosylpetunidin & $\mathbf{P t}$ & 17.88 & & \\
\hline 5 & 3-O-Rutinosylpeonidin & Po & 19.45 & 0.45 & 0.70 \\
\hline 6 & 3-O-Rutinosylmalvidin & $\mathrm{Ma}$ & 20.55 & 0.57 & 0.89 \\
\hline
\end{tabular}

Table 4. Anthocyanins content (\%) in wild Musa species accession based on HPLC analysis

\begin{tabular}{lcccccc}
\hline Samples & $\%$ \%e & $\%$ Cyd & $\%$ Cy & $\% P t$ & $\%$ Po & $\%$ Ma \\
\hline RI & 0.01 & 1.47 & 14.63 & 1.06 & 32.73 & 50.10 \\
IPTJ & 0.10 & 3.57 & 20.04 & 4.40 & 35.48 & 36.41 \\
KRA & 0.00 & 1.13 & 20.09 & 1.62 & 59.50 & 17.66 \\
BD1 & 0.00 & 0.51 & 22.16 & 3.75 & 32.45 & 41.13 \\
BD2 & 0.00 & 3.73 & 37.02 & 1.00 & 39.42 & 18.83 \\
BC1 & 0.00 & 1.19 & 21.13 & 3.16 & 33.19 & 41.33 \\
BC3 & 0.00 & 13.34 & 14.17 & 18.18 & 2.79 & 51.52 \\
Segun & 0.00 & 0.06 & 8.22 & 2.73 & 52.10 & 36.89 \\
Sintok & 0.10 & 0.88 & 9.98 & 2.90 & 42.97 & 43.17 \\
Rangis & 0.00 & 1.99 & 10.71 & 3.51 & 34.10 & 49.69 \\
Perak & 0.00 & 1.11 & 11.70 & 0.00 & 38.35 & 48.84 \\
PPC & 0.08 & 1.69 & 18.03 & 2.83 & 10.79 & 36.58 \\
J-4 & 0.00 & 2.81 & 14.83 & 3.73 & 12.86 & 35.77 \\
Flava & 0.00 & 0.18 & 0.07 & 0.10 & 1.85 & 2.74 \\
Gala & 0.00 & 14.48 & 84.63 & 0.00 & 0.89 & 0.00 \\
BC2 & 0.00 & 6.62 & 0.40 & 7.93 & 8.18 & 25.30 \\
\hline De $=$ Delphinidin & Cyd $=$ Cyanidin derivative & & Cy $=$ & Cyanidin \\
Pt = Petunidin & Po & Peonidin & & Ma $=$ & Malvidin
\end{tabular}


The data matrix of relative amounts of floral anthocyanins expressed by percentages for Musa samples (Table 4) was then subjected to cluster and principal component analysis (PCA). Cluster analysis was conducted by using Ward's method with squared euclidean distance (Fig 1). Four principal components with eigenvalues more than 1.0 were obtained, which could explain $99.89 \%$ for the total variation in the data matrix. Thus all samples were grouped into four based on cluster and principal component analysis (Table 5).

Rescaled Distance Cluster Combined

\begin{tabular}{ccccccc}
\hline $\begin{array}{c}\text { Compound } \\
\text { number }\end{array}$ & Assigned structure & Abbreviation & $\begin{array}{c}\text { HPLC retention } \\
\text { times (min) }\end{array}$ & $\begin{array}{c}\text { Observed Rf } \\
\text { values } \\
\text { Syst.1 }\end{array}$ & Syst.2 \\
\hline 1 & 3-O-Rutinosyldelphinidin & De & 12.43 & 0.32 & \\
2 & Cyanidin derivative & Cyd & 13.30 & & \\
3 & 3-O-Rutinosylcyanidin & Cy & 15.51 & 0.26 & 0.50 \\
4 & 3-O-Rutinosylpetunidin & Pt & 17.88 & 0.45 & 0.70 \\
5 & 3-O-Rutinosylpeonidin & Po & 19.45 & 0.57 & 0.89 \\
6 & 3-O-Rutinosylmalvidin & Ma & 20.55 & 0.57 \\
\hline
\end{tabular}

able 4. Anthocyanins content (\%) in wild Musa species accession based on HPLC analysis

\begin{tabular}{lcccccc}
\hline Samples & $\%$ De & \%Cyd & \%Cy & \%Pt & \%Po & $\%$ Ma \\
\hline RI & 0.01 & 1.47 & 14.63 & 1.06 & 32.73 & 50.10 \\
IPTJ & 0.10 & 3.57 & 20.04 & 4.40 & 35.48 & 36.41 \\
KRA & 0.00 & 1.13 & 20.09 & 1.62 & 59.50 & 17.66 \\
BD1 & 0.00 & 0.51 & 22.16 & 3.75 & 32.45 & 41.13 \\
BD2 & 0.00 & 3.73 & 37.02 & 1.00 & 39.42 & 18.83 \\
BC1 & 0.00 & 1.19 & 21.13 & 3.16 & 33.19 & 41.33 \\
BC3 & 0.00 & 13.34 & 14.17 & 18.18 & 2.79 & 51.52 \\
Segun & 0.00 & 0.06 & 8.22 & 2.73 & 52.10 & 36.89 \\
Sintok & 0.10 & 0.88 & 9.98 & 2.90 & 42.97 & 43.17 \\
Rangis & 0.00 & 1.99 & 10.71 & 3.51 & 34.10 & 49.69 \\
Perak & 0.00 & 1.11 & 11.70 & 0.00 & 38.35 & 48.84 \\
PPC & 0.08 & 1.69 & 18.03 & 2.83 & 10.79 & 36.58 \\
J-4 & 0.00 & 2.81 & 14.83 & 3.73 & 12.86 & 35.77 \\
Flava & 0.00 & 0.18 & 0.07 & 0.10 & 1.85 & 2.74 \\
Gala & 0.00 & 14.48 & 84.63 & 0.00 & 0.89 & 0.00 \\
BC2 & 0.00 & 6.62 & 0.40 & 7.93 & 8.18 & 25.30 \\
\hline Je = Delphinidin & Cyd = Cyanidin derivative & & Cy & $=$ & Cyanidin \\
't $=$ Petunidin & Po & $=$ Peonidin & & Ma $=$ & Malvidin
\end{tabular}

Figure 1. Cluster analysis of Musa accessions based on anthocyanins using Ward's method. All Musa acuminata accessions were grouped into two major clusters except for the sample Flava. The highland banana BC3 was well separated from other Musa acuminata accessions. Similarly, Gala (Musa balbisiana) and BC2 (Musa violascens) were also well separated. 
Gala was completely separated from the Musa acuminata and Musa violascens by larger values in the score for third PC (Table 5), and is characterized by the larger accumulation of cyanidin (84.63\%) followed by cyanidin derivative (Table 4). Factor loadings of pigments cyanidin and cyanidin derivative for the third PC were also great (Table 6).

Highland Musa acuminata sample (BC3) was completely separated from other samples of the species gaining the highest score for the second PC (Table 5), for which factor loadings of pigment petunidin were great (Table 6), whereas the cyanidin derivative was also accumulated in the highest percentage compared to other accessions of M acuminata samples. However, this sample showed the lowest peonidin compared to other samples of the same species.

Table 5. Principal component scores of Musa samples

\begin{tabular}{lllrr}
\hline \multicolumn{1}{c}{ Sample } & PC1 & PC2 & PC3 & PC4 \\
\hline Group I. & & & & \\
BC1 & 0.980 & 0.146 & 0.132 & -0.019 \\
BDI & 0.973 & 0.153 & 0.160 & -0.020 \\
BD2 & 0.968 & 0.243 & -0.034 & 0.028 \\
IPTJ & 0.973 & 0.221 & 0.047 & 0.051 \\
J-4 & 0.957 & 0.234 & -0.156 & 0.069 \\
Kra & 0.912 & 0.235 & 0.303 & 0.143 \\
Perak & 0.997 & 0.003 & -0.047 & -0.058 \\
PPC & 0.964 & 0.256 & -0.065 & 0.035 \\
Rangis & 0.995 & -0.094 & -0.011 & -0.036 \\
RI & 0.993 & -0.046 & 0.073 & -0.074 \\
Segun & 0.918 & 0.217 & -0.325 & 0.060 \\
Sintok & 0.982 & 0.079 & -0.172 & 0.009 \\
Group II. & & & & -0.043 \\
BC2 & 0.966 & -0.203 & -0.141 & \\
Group III. & & & & 0.120 \\
Flava & 0.773 & -0.620 & 0.055 & \\
Group IV. & & & & 0.081 \\
BC3 & 0.603 & -0.631 & 0.481 & \\
Group V. & & & & 0.042 \\
Gala & -0.136 & 0.606 & 0.781 & \\
\hline
\end{tabular}

Table 6. Factor loadings of respective pigments exiting in Musa species to principal components

\begin{tabular}{lrrrr}
\hline \multicolumn{1}{c}{ Component } & PC1 & PC2 & PC3 & PC4 \\
\hline Delphinidin & -0.362 & 0.069 & 0.328 & -0.283 \\
Cyanidin derivative & 0.910 & 0.243 & 0.231 & 0.182 \\
Cyanidin & 0.647 & -0.529 & 0.886 & 0.424 \\
Petunidin & 0.321 & 0.903 & 0.037 & 0.016 \\
Peonidin & -0.812 & -0.251 & 0.087 & 0.398 \\
Malvidin & -0.529 & 0.666 & 0.080 & 0.425 \\
\hline
\end{tabular}


Musa violascens (BC2) showed a very different chemotype and a number of peaks produced varied from other two species samples greatly. The anthocyanins detected in the other two species were present in a very small percentage. Similarly, Musa acuminata sample Flava was observed with a very small percentage of the six anthocyanins detected.

Twelve samples were classified into first group gaining the largest scores for first principal component (Table 5), for which factor loadings of peonidin, cyanidin and malvidin were great (Table 6), whose anthocyanin composition of the bracts seems to be the standard of the Musa acuminata samples. The only variation observed among these samples was the ratio of peonidin and malvidin. Different from the highland banana samples with a large accumulation of peonidin whereas, cyanidin derivative and petunidin were present in a very low percentage (Table 4).

As shown in Fig (1) and Table (5), three groups of Musa acuminata were readily distinguishable. Flava diverges from other two groups of samples by'the absence of anthocyanins, as it had yellow color bracts i.e. acyanic bracts. Highland banana sample BC3, had a very distinct deep chocolate brown color of the male bracts, and was characterized with accumulation of the simple anthocyanins in this subspecies, whose total composition could be described as 3 - rutinosides of cyanidin derivative (Cyd), 3 - rutinosides of petunidin (Pt) (Table 4). These anthocyanins were mainly present in this sample. However, a low percentage of peonidin was detected. The remaining Musa acuminata accessions belonging to lowlands of Malaysia showed similar anthocyanins and their bract color ranged from red and purple. Similarly, all Musa acuminata accessions suggested a very high ability of methylation of anthocyanidins compared to Gala (M. balbisiana).

\section{DISCUSSIONS}

Many cytotaxonomists have argued that a unique chemical feature should merit the same importance as an unusual leaf shape or an odd chromosome number in determining plant relationships. They have shown that chemical characters correlate in genetic surveys satisfactorily with one or more biological characters. Harborne (1967) made a survey of flavonoid pigments in $45 \%$ of the genera in the family Gesneriaceae against the classification proposed based on morphological grounds. The differences at the subfamily level indicated a correlation between anthocyanin chemistry and plant geography.

Anthocyanin components were found to be very stable and the chromatograms obtained were highly reproducible under the same conditions. Although the yield of anthyocyanins in Musa accessions may vary, the qualitative aspect of it remains relatively consistent, thus making them good taxonomic characters. More than nine peaks were observed in most of the samples, indicating that at least similar number of components were present. However, in the present study only six components were recognized following Horry and Jay (1988 a,b). From the chromatograms, all the Musa species can be identified. Musa acuminata accessions were characterized 
with partially methylated anthocyanins based on cyanidin - delphinidin mixtures i.e. mixtures of cyanidin, peonidin, delphinidin, petunidin and malvidin in different proportions. Flava, a yellow bracted accession, did not show significant amount of anthocyanins. Simmonds (1954) reported that Musajlava Ridl. from Malaysia was merely a yellow bracted form of Musa acuminata and the yellowness was due a single recessive gene and Flava was a good example of acyanic bracts (bracts without anthocyanins). Musa violascens was observed with a very different chromatogram than the other two and it showed a very little amount of six anthocyanins as observed in remaining species. The anthocyanin variation observed in this species could also be related to the light pink bract color compared to the red purple color observed in other species. Anthocyanin variation among these species was further suggested by their different pollinators. Musa violascens were reported to be bird pollinated whereas $M$. acuminata and $M$. balbisiana flowers were pollinated by bats (Simmonds 1962; Argent 1976).

Horry and Jay (1988 a, b) described a schematic sequence for anthocyanin biosynthesis in Musa. They described that cyanidin is the first and simplest element in a sequence of hydroxylations whereas methylations ending at malvidin. Cyanidin or peonidin always occur, suggesting that cyanidin is an obligatory intermediate. We have observed high levels of cyanidin and peonidin in all Musa acuminata accessions except for the highland banana form BC3. BC3 was observed to have a very low level of peonidin whereas a new compound Cyd (cyanidin derivative) was deposited in high percentage compared to all other accessions. This compound was also reported by Horry and Jay (1988a, b) in Musa balbisiana and suggested a different biochemical pathway. It is found to be an interesting marker for highland banana forms, therefore, it merits further characterization. A similar situation was observed for petunidin, which was accumulated in a very high percentage in BC3. Horry and Jay (1988b) suggested that a low level of petunidin and the fact that it never accumulates without malvidin, leads to the assumption of O-methylations. These two compounds were observed in high percentage in all lowland banana samples except in BC3.

In terms of evolutionary significance of anthocyanins, there is a trend for delphinidin to be replaced by cyanidin, which is considered to be the most primitive pigment. Therefore, O-methylation represents an advanced feature in comparison with simple hydroxylation (Harborne 1977). All lowland Musa acuminata accessions were observed with low levels of petunidin compared to the high percentage of malvidin, suggesting an advanced feature of anthocyanins whereas it was different in highland banana accession showing a high percentage of petunidin. Simmonds (1962); Horry and Jay (1988a,b) suggested that chemotype of Musa balbisiana is fairly unique and possesses no "advanced" anthocyanin forms and that environmental constraints were not so extreme that specialized biochemical pathways were needed to be developed in the evolution of this species. On the other hand, Musa acuminata was found to possess a broad range of chemotypes, indicating the presence of a more diversified metabolism. This suggests that Musa acuminata has undergone more evolutionary changes than Musa balbisiana. 
Cluster analysis and principal component analysis (PCA) produced five groupings in Musa acuminata accessions based on anthocyanins. The highland banana BC3 showed completely different anthocyanin patterns and was well separated from the remaining accessions. It was also observed to be morphologically distinct. All lowland Musa acuminata accessions showed variation mainly in the proportion of the peonidin and malvidin. Malvidin was predominant in RI, IPTJ, BD1, BC1, Segun, Sintok, Rangis, and Perak whereas peonidin in Kra, BD2, PPC and J-4, respectively. The discrimination found among accessions, therefore, was mainly due to the ratios of these two anthocyanins whereas overall anthocyanin distribution was found similar among these accessions and the mean chemotype proceeded from a simple readjustment of the metabolic balance between methylated anthocyanins (Horry and Jay 1988a).

The relationship revealed by the anthocyanin fingerprints could be compared with the geographical distribution and origin of these accessions. Musa acuminata accessions were collected from lowlands and highlands. A large variation in the anthocyanin composition was observed for the highland banana form (ssp. truncatd) compared to lowland samples. Northern (ssp. siamea) and central Malayan (ssp. malaccensis) region samples were observed with similar anthocyanin compositions. Therefore, the geographical origin of lowland accessions had little effect upon the anthocyanins metabolism. Whereas the highland banana was different in ecology and geography and also showed distinct anthocyanin pattern.

Anthocyanin data further supported the two groupings of the Musa acuminata i.e. lowland (ssp. malaccensis) and highland (ssp. truncatd) as suggested by Hari (1968) and Shepherd (1988).

\section{ACKNOWLEDGEMENTS}

The work was supported by a grant from the Ministry of Science and Technology and Environment, Malaysia. We also would like to thank Mr. Gopal Krishnen and Mr. Asokan for their kind help in running the HPLC system analysis.

\section{REFERENCES}

Anderson, O. M. and G. W. Francis. 1985. Simultaneous analysis of anthocyanins and anthocyanidins on Cellulose thin layers. J. Chromatography. 318,450-454.

Argent, G. C. G. 1976. The Wild Bananas of Papua New Guineae. Notes of Royal Botanical Garden, Edinburgh 35: 77-114.

Cheesman, E. E. 1947. Classification of the Bananas. Kew Bull. 3, 11-28.

Hari, P. C. 1968. Bract imbrication as a taxonomic character in Musa acuminata. Trop. Agric. Trinidad. Vol. 45, No. 2,99-108.

Harborne, J.B. 1967. Comparative biochemistry of the flavonoids. Academic Press. London.

Harborne, J. B. 1977. Flavonoids and the evolution of angiosperms. Biochem. Sys. Ecol. 5, 5-22 
Horry, J. P. and M. Jay. 1988a. Distribution of anthocyanins in wild and cultivated banana varieties. Phytochemistry. 27, 2667-2672.

Horry, J. P. and M. Jay. 1988b. An evolutionary background of bananas as deduced from flavonoids diversification. In: (Jarret, R. L. ed.). Identification of Genetic Diversity in the genus Musa, INIBAP. Montpellier, France, p. 158-165.

Shepherd, K. 1988. Observations on Musa taxonomy. In: (Jarret, R. L. ed.). Identification of Genetic Diversity in the genus Musa, INIBAP. Montpellier, France, p. 158-165.

Simmonds, N. W. 1954b. Anthocyanins in bananas. Nature, 173,402. Simmonds, N.

W. 1954c. Anthocyanins in banana. Ann. Bot. Lond. 18, 471-82. Simmonds, N. W.

1955. Wild bananas in Malaya. Malayan Nature Journal. 10, 1-8. Simmonds, N. W.

1962. The evolution of the bananas. London. UK. Longman. 